\title{
Las huellas lingüísticas de la teoría de la mente: intersubjetividad y enunciación en el trastorno por déficit de atención/hiperactividad
}

\author{
B. Gallardo-Paúls
}

\author{
LAS HUELLAS LINGÜÍSTICAS DE LA TEORÍA DE LA MENTE: INTERSUBJETIVIDAD \\ Y ENUNCIACIÓN EN EL TRASTORNO POR DÉFICIT DE ATENCIÓN/HIPERACTIVIDAD
}

\begin{abstract}
Resumen. Introducción. Las pruebas utilizadas habitualmente para analizar si un sujeto ha desarrollado capacidades mentalistas e intersubjetivas (teoría de la mente) se ciñen a tareas de evaluación de la comprensión, sin considerar las huellas activas, expresivas, de esas capacidades. Desarrollo. Tomando como marco la teoría pragmática de la enunciación de Benveniste, analizamos textos escritos por niños con diagnóstico de trastorno por déficit de atención/hiperactividad (TDAH) para verificar la presencia de marcas formales de la enunciación. Distinguimos entre las marcas explícitas en el texto (sintagmas verbales o nominales que refieran estados mentales y de dicción) y las marcas de textos incrustados (diálogos, alusiones a textos ajenos, polifonía textual). El corpus de datos utilizado para este análisis consiste en 170 textos narrativos y argumentativos escritos por niños de 9 a 11 años, un subgrupo de los cuales presenta diagnóstico de TDAH tipo combinado. Conclusiones. La teoría de la enunciación proporciona el marco adecuado para estudiar la manifestación expresiva, activa, de la capacidad intersubjetiva del niño. [REV NEUROL 2008; 46 (Supl 1): S29-35]

Palabras clave. Déficit lingüístico. Narración y argumentación. Pragmática textual. Teoría de la mente. Trastorno por déficit de atención/hiperactividad.
\end{abstract}

\section{INTRODUCCIÓN}

La capacidad que Premack y Woodruff [1] definieron como 'teoría de la mente', a propósito de la chimpancé Sara y sus reacciones ante vídeos de resolución de problemas, se refiere a una habilidad cognitiva que la psicología había descrito con anterioridad mediante conceptos muy diversos. Pensemos, por ejemplo, en la inteligencia social de Thorndike, los procesos de autocentración y descentración descritos por Piaget [2], la importancia dada por Vigotsky a la interacción comunicativa en el desarrollo cognitivo [3], o las modalidades de representación enactiva, icónica y simbólica propuestas por Bruner [4,5]. Con todo, la nueva etiqueta gozó de rápida aceptación y dio actualidad al estudio de una serie de capacidades complejas (TirapuUstárroz et al hablan directamente de 'cajón de sastre' [6]), que permiten al individuo decodificar y predecir de manera verosímil el estado mental ajeno.

\footnotetext{
Aceptado: 14.01.08.

Lingüística General. Facultad de Filología, Traducción y Comunicación. Universitat de València. Valencia, España.

Correspondencia: Dra. Beatriz Gallardo-Paúls. Lingüística General. Facultad de Filología, Traducción y Comunicación. Universitat de València. Avda. Blasco Ibáñez, 32, 5. ․ E-46010 Valencia. Fax: +34 963864 778. E-mail: beatriz.gallardo@uv.es

Trabajo realizado en el marco de los proyectos de investigación financiados 'Pragmática textual en la afasia y otras patologías del lenguaje: elementos de coherencia, cohesión y conexidad' (Universitat de València, ref. UV-AE2007) y 'Protocolo de análisis pragmasintáctico. Un estudio de corpus oral' (MEC, ref. HUM2007-66074-C02-02).

Los datos se han obtenido en el marco de diversos convenios de colaboración firmados por el área de lingüística general de la Universitat de València. La mayor parte de los textos narrativos procede del Centro CADAN de Atención al Neurodesarrollo de Badajoz, dirigido por el Dr. J. Vaquerizo. Los textos argumentativos han sido escritos por pacientes del Instituto Valenciano de Neurología Pediátrica, dirigido por el Dr. F. Mulas. Los grupos control pertenecen a diversos colegios públicos de la ciudad de Valencia. Agradecemos igualmente la colaboración de M.J. Baranda y E. Ponce. El proceso de elaboración del corpus y la investigación continúan, con la finalidad de poder realizar un protocolo de evaluación y seguimiento basado en texto escrito.

(C) 2008, REVISTA DE NEUROLOGÍA
}

La rápida aplicación, en los años ochenta, de la teoría de la mente a ámbitos clínicos como el autismo, el síndrome de Asperger, el del cromosoma $X$ frágil o el daño cerebral, trata de evaluar la existencia de tales capacidades en los sujetos a partir de habilidades receptivas, pasivas, de comprensión. Las conocidas tareas de falsa creencia, sobre la canica escondida, el chocolate guardado o la caja de 'lacasitos' [7-19] examinan la capacidad infantil de atribuir creencias a terceras personas en situaciones de discordancia con la realidad. Pese a interesantes diferencias interculturales, la mayoría de los estudios concluye que aproximadamente a partir de los cuatro años el niño es capaz de asumir que la conducta de los demás no se basa sólo en la realidad objetiva, sino en las propias creencias y opiniones (las cuales pueden estar equivocadas por falta de datos).

La realización correcta de estas tareas exige al niño admitir dos posibles representaciones de una misma realidad, separar la propia creencia de la ajena, un proceso que, tenga o no base en un mecanismo innato [20,21], se construye necesariamente a partir de la interacción real y la experiencia personal; de ahí que prefiramos el término de 'capacidad intersubjetiva' al de 'teoría de la mente', en la línea de autores como Hobson [22] o Rondal [23].

En este trabajo nos planteamos cuál es la dimensión activa, expresiva, de esa misma capacidad intersubjetiva, es decir, cuáles son las huellas lingüísticas concretas que nos permiten afirmar su existencia en el habla de una persona. Para ello nos basaremos en la teoría pragmática de la enunciación, propuesta por el lingüista Benveniste [24], según la cual en cada acto de habla los hablantes nos adueñamos de la lengua para construir nuestros mensajes (nuestros 'enunciados'), pero dejando siempre en ellos marcas formales de esa situación que integramos hablantes y oyentes, es decir, del marco comunicativo (la 'enunciación'); la identificación de este tipo de rasgos lingüísticos (a veces, palabras concretas, pero otras veces, rasgos morfosintácticos) nos da información sobre los hablantes, su intención comunicativa o su actitud frente a los receptores o el propio mensaje. Utilizar la pragmática como marco teórico nos permite dar coherencia global a las investigaciones habituales de la teoría de la mente, pues 
podemos utilizar una teoría unificada $[25,26]$ para explicar la adecuada comprensión de todos los fenómenos de inferencia, como ironía, metáfora, inconveniencia, indiscreción o descortesía verbal, etc., evaluadas habitualmente por separado, mediante tareas de historias extrañas y falsas creencias [27-32].

\section{OBJETIVO Y METODOLOGÍA}

Nuestro objetivo es rastrear la utilización de marcas formales de intersubjetividad en textos escritos por niños con trastorno por déficit de atención/hiperactividad (TDAH). Puesto que los procesos de socialización e interacción lingüística que sirven de marco para el desarrollo de esta capacidad [33-35] exigen la activación simultánea de expresión y comprensión verbal, creemos que es importante establecer marcas expresivas que indiquen la existencia de dicha capacidad en el propio niño (no sólo en los adultos que se relacionan con él). Con este fin, para cada uno de los textos diferenciamos dos niveles:

- El nivel del texto escrito por el niño, es decir, las manifestaciones de que un niño está redactando cierto texto narrativo o argumentativo (enunciación).

- Un nivel incrustado, es decir, situaciones enunciativas que el niño incluye en su texto (enunciado), como diálogos de personajes, mensajes, anécdotas que ejemplifican las argumentaciones, etc.

Como hemos señalado, el autor del texto deja 'huellas de la enunciación' en ambos niveles, es decir, marcas formales que nos informan sobre él mismo y su actitud hacia lo que dice; la utilización de estas marcas le exige el desarrollo de una capacidad intersubjetiva. Podemos agrupar estos rasgos de intersubjetividad en las siguientes categorías:

- Informaciones cognitivas explícitas, es decir, que revelan creencias, sentimientos o intenciones, tanto del autor del texto como de sus personajes. Identificamos aquí:

a) Verbos mentalistas (creer, saber). Los personajes (tanto de las narraciones como de los ejemplos y anécdotas incluidos en las argumentaciones) se definen por creencias, opiniones, intenciones o sentimientos.

b) Sintagmas nominales y expresiones relacionadas, que completan las acciones de los personajes o matizan el sentido de las acciones.

- Argumentaciones mentalistas: se trata de argumentaciones justificativas y explicativas que no se refieren al tema tratado o a la historia construida, sino al propio proceso de redacción; su utilización destaca especialmente en los textos narrativos, que obligan al niño a construir un universo discursivo diferente al de la redacción.

- Marcas de intertextualidad y polifonía textual, es decir, la existencia de varias voces en el interior del texto:

a) Utilización de verbos de dicción que introducen voces en el texto.

b) Alusiones a las personas de la enunciación, el 'yo' que escribe y el 'tú/vosotros' que leerá; el niño demuestra su conciencia discursiva y emerge en su propio texto, implicando directamente a su receptor.

\section{DATOS DEL ANÁLISIS}

Los datos de partida utilizados en este trabajo consisten en diversos tipos de textos elaborados por niños con TDAH. En la ta-
Tabla I. Textos del corpus: argumentación (AEP) y narración (NEP) escrita planificada.

\begin{tabular}{lcccc}
\hline & \multicolumn{2}{c}{ Textos del grupo TDAH } & \multicolumn{2}{c}{ Textos del GRC } \\
\cline { 2 - 5 } & NEP & AEP & NEP & AEP \\
\hline 9 años & 6 & 2 & 26 & 24 \\
\hline 10 años & 10 & 6 & 30 & 23 \\
\hline 11 años & 4 & 4 & 20 & 15 \\
\hline Total & 20 & 12 & 76 & 62 \\
\hline
\end{tabular}

GRC: grupo control; TDAH: trastorno por déficit de atención/hiperactividad.

bla I detallamos datos sobre los textos del grupo TDAH y el grupo control (GRC).

Hemos planteado dos modalidades diferentes de redacción [36], porque creemos que el tipo de texto puede determinar la aparición preferente de algunas de estas categorías. Aunque la bibliografía clínica ha prestado notable interés a la comprensión y elaboración de historias por parte de niños con TDAH [37-45] o con otras alteraciones, la atención a otro tipo de estructuras textuales es completamente nula. Para este estudio hemos elaborado un corpus de textos escritos que incluye narraciones y argumentaciones, porque creemos, siguiendo a Bruner, que se trata de dos modelos textuales que corresponden a dos diferentes estrategias cognitivas, por lo que su análisis debe ser necesariamente complementario.

En los textos narrativos -narraciones escritas planificadas (NEP)-, se le pide al niño que redacte cierta historia a partir de unos elementos cerrados (tres personajes, un objeto mágico, un punto de partida, un lugar, un objetivo), según el conocido modelo de La fábrica de cuentos [46-47]. Para la obtención de textos argumentativos -argumentaciones escritas planificadas (AEP) hemos diseñado varias tareas, algunas de ellas basadas en imágenes, que solicitan al niño motivos, justificaciones y argumentaciones (Fig. 1).

Para cada texto hemos realizado análisis iniciales que tienen en cuenta la complejidad sintáctica (con medidas referidas a longitudes medias de oración y proposición, tipos de subordinación y grado de incrustación subordinada) y lo que podríamos llamar la densidad textual de cada escrito; en los textos narrativos este rasgo se refiere al número de sucesos incluidos en la trama, la definición de los personajes, lugares y tiempos, o la existencia de sucesos incompletos; en los textos argumentativos, la densidad textual incluye básicamente el número y clasificación de las argumentaciones aducidas por el niño para cada norma señalada. Además, estos análisis pragmáticos se completan con la identificación de marcas formales de la enunciación, que desarrollamos específicamente en el próximo apartado.

La primera diferencia evidente entre los textos narrativos y argumentativos se refiere a la extensión, según refleja la tabla II, lo que parece indicar que el niño asume el papel redactor con más seguridad en el texto narrativo, posiblemente porque es un tipo de texto con el que está muchísimo más familiarizado. Como refleja la tabla II, los textos TDAH son más breves que los del GRC en todas las medidas.

Por lo que se refiere a la sintaxis, no cabe señalar diferencias notables con el GRC ni en el nivel medio de incrustación subordinada (1,8 para el grupo TDAH y 2,2 para el GRC), ni en 


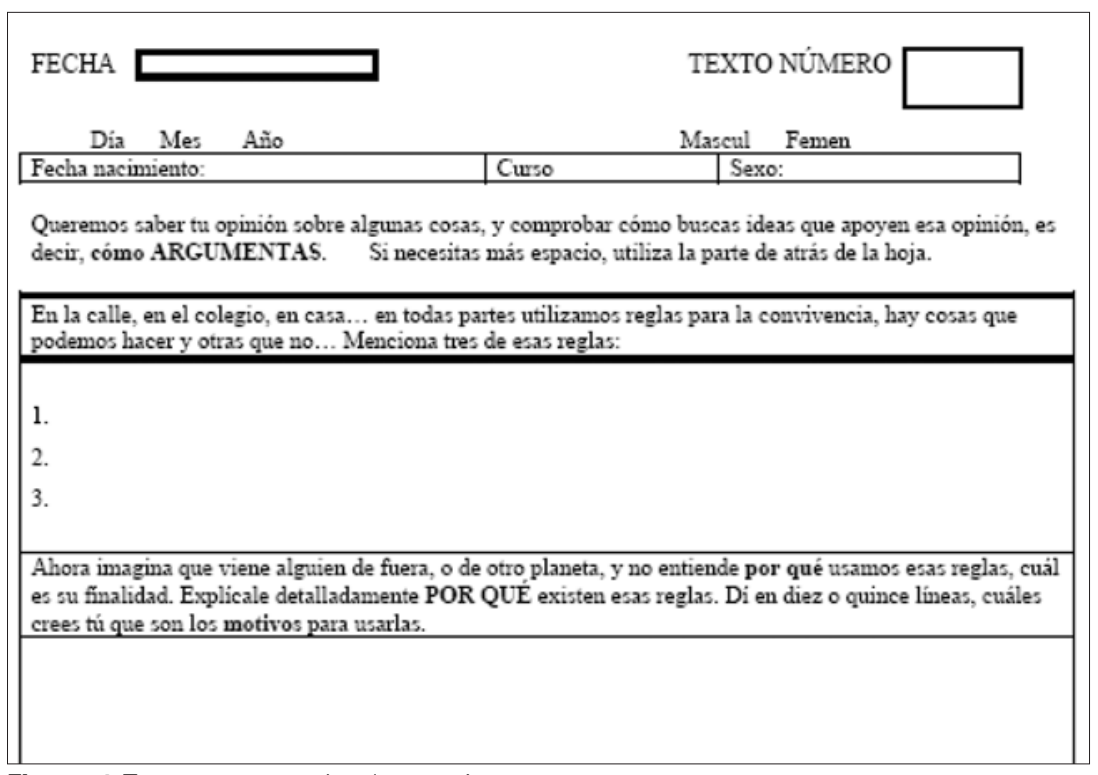

Figura. 1. Tarea argumentativa: 'normas'.

Tabla II. Longitudes medias de ambos corpus y ambos tipos de texto: separación por edades.

\begin{tabular}{|c|c|c|c|c|c|c|c|c|c|}
\hline & & \multicolumn{4}{|c|}{ Textos del grupo TDAH } & \multicolumn{4}{|c|}{ Textos del GRC } \\
\hline & & 9 a & $10 \mathrm{a}$ & $11 \mathrm{a}$ & TDAH & $9 a$ & $10 a$ & $11 \mathrm{a}$ & GRC \\
\hline \multirow[t]{2}{*}{ Palabras } & NEP & 66,7 & 103,5 & 124,3 & 98,2 & 155,3 & 198,6 & 180,2 & 178 \\
\hline & AEP & 24 & 54,8 & 76,3 & 51,7 & 78,8 & 115,5 & 120,6 & 104,9 \\
\hline \multirow[t]{2}{*}{ Oraciones } & NEP & 4,7 & 4,4 & 4,3 & 4,5 & 6,9 & 12,7 & 12,2 & 10,6 \\
\hline & AEP & 4 & 4,5 & 4,5 & 4,3 & 6 & 7,1 & 8 & 7 \\
\hline \multirow[t]{2}{*}{ Proposiciones } & NEP & 12,8 & 18,9 & 22 & 17,9 & 28,5 & 37,6 & 32,5 & 32,9 \\
\hline & AEP & 5,5 & 11,3 & 16 & 10,9 & 15,7 & 29,4 & 23 & 22,7 \\
\hline $\begin{array}{l}\text { Subordinadas } \\
\text { por oración }\end{array}$ & & 1,1 & 1,2 & 1,3 & 1,2 & 0,9 & 1,3 & 1,3 & 1,2 \\
\hline Sucesos & NEP & 2,6 & 4,5 & 4,8 & 3,9 & 4,5 & 4,7 & 5,8 & 5 \\
\hline Justificaciones & AEP & 2,5 & 3,2 & 3,8 & 3,2 & 4,8 & 5,2 & 5,7 & 5,2 \\
\hline
\end{tabular}

a: años; AEP: argumentación escrita planificada; GRC: grupo control; NEP: narración escrita planificada; TDAH: trastorno por déficit de atención/hiperactividad.

la cantidad media de subordinadas por oración (1,2 subordinadas en el grupo TDAH y 1,1 en el GRC); en este sentido, constatamos que para ambos corpus las argumentaciones se prestan más a la aparición de subordinadas sustantivas, en la medida en que se nombran acciones (sustantivas de infinitivo o de complemento con perífrasis de obligación), y de subordinadas adverbiales (muy especialmente condicionales, que especifican circunstancias que justifican el argumento, y causales, que explicitan las justificaciones), mientras que las subordinadas adjetivas tienen mucha mayor funcionalidad en las narraciones, especialmente en la descripción de personajes.

En el ámbito pragmático textual existen diferencias leves entre los dos grupos. Como hemos dicho, las categorías textuales analizadas se refieren a la densidad narrativa desarrollada por el niño (personajes, tiempos, lugares, complicaciones y resolucio- nes en los textos NEP) y a su capacidad argumentativa (justificaciones y conclusiones en los textos AEP). La complejidad narrativa fundamental, derivada del número de complicaciones y resoluciones de la historia, parece comparable entre ambos grupos (un poco menor en el grupo TDAH, especialmente en la franja de menos edad), si bien el grupo TDAH muestra mayor frecuencia de sucesos incoherentes que se explican especialmente por el uso confuso de conectores [48], dificultades que pueden vincularse a problemas con la memoria de trabajo $[6,49]$. Por otro lado, la ligera diferencia que se aprecia en cuanto al número de justificaciones que incluye cada niño en su texto (5,2 en GRC y 3,2 en TDAH) puede deberse, al menos parcialmente, a diferencias en los tipos de tarea. Respecto al tipo de argumentos utilizados por los niños de ambos grupos, encontramos los tres tipos básicos -codificamos los ejemplos con estos datos: letra inicial que indica el corpus TDAH (T) o el GRC (C), número del texto, edad y sexo; en todos los textos hemos corregido ortografía, acentuación y puntuación-:

- Estrategias argumentativas de sanción, es decir, de premio o castigo (69 argumentos en el total de la muestra, de los cuales sólo dos son de premio): 'Hay que cumplir unas normas para que no te multen' (T021, 10:10, M), 'no hay que pegar, porque si nos pegamos nos castigan y si son muchas veces nos pueden abrir un expediente' (C470, 9:05, M), 'Si no respetas a nadie, no tendrás amigos, nadie te querrá ni te respetarán, y eso, en ocasiones, no es, que digamos, muy beneficioso' (T024, 12:00, M).

- Estrategias lógicas o de instrucción (121 argumentos en total, que representan un $28,2 \%$ en el grupo TDAH y un $34,8 \%$ en el GRC); incluimos aquí las argumentaciones referidas a consecuencias más o menos objetivas, y a las justificaciones realizadas mediante ejemplos, que son bastante numerosas: 'es como si tú le pegaras a él y él no te ha hecho nada' (T002, 10:04, M).

- Estrategias falaces, un total de 165, entre las que encontramos 56 argumentos circulares o incoherentes y 109 argumentos moralistas. Analizar el tipo de argumentos que utilizan los niños de nuestro corpus excede los propósitos de este trabajo y es el objeto de otra investigación en curso. Señalamos tan sólo que, igual que el niño aprende a narrar gracias a su conocimiento de historias ajenas (planificadas/literatura o espontáneas/relatos orales), su manera de argumentar se nutre necesariamente de los argumentos, explicaciones y justificaciones que recibe del entorno; y en este sentido, tratándose de niños entre 9 y 11 años, resulta interesante el marcado predominio de los argumentos moralistas y catastrofistas, con claros matices ecoicos respecto al discurso adulto: 'Hay que respetar a los demás porque así los demás te respetan a ti' (T005, 11:11, M). Hemos incluido entre las falacias mora- 
listas todas las que enmascaran falta de argumentos reales o revelan visiones catastrofistas: 'Si no hacemos unas ciertas normas nos podemos volver locos, sin normas todo esto parecería un zoo' (C485, 9:05, M); 'Si todo lo que te encuentras lo rayas al final todo estaría hecho un asco, sería un caos y todos estarían peleándose por rayar algo' (C588, 10:03, F).

\section{MARCAS DE INTERSUBJETIVIDAD}

En este apartado prestaremos atención separadamente a los elementos que hemos identificado como huellas explícitas de capacidad intersubjetiva; nos situamos en el ámbito de la pragmática enunciativa, es decir, atendemos a los elementos del lenguaje que vienen determinados por el uso que hace cierto hablante, guiado por una intención comunicativa concreta, que busca causar cierto efecto en su interlocutor y transmitirle cierto significado. La idea básica es que todas las emisiones verbales (orales o escritas) tienen huellas materiales, formales, del acto de habla en que se producen.

\section{Informaciones cognitivas explícitas}

Incluimos en este apartado aquellos elementos del léxico que suponen una capacidad de mentalizar o considerar actividades cognitivas propias o en terceras personas [50-52], diferenciando dos categorías distintas:

- Aparición de verbos mentalistas que refieren acciones relacionadas: pensar, considerar, creer, respetar, culpar... En las narraciones, los niños caracterizan a sus personajes a partir de sus creencias, intenciones, etc.: 'Volvía cansada cuando, de pronto, frente a su casa, encontró una huella misteriosa. Quiso taparla, pero no pudo. Quiso huir, pero sus piernas no reaccionaban. Quiso gritar, pero sus labios no se lo permitían' (C410, 10:02, M).

- Aparición de otras expresiones mentalistas, pertenecientes a otras categorías morfosintácticas: 'deseo, verdad, agradecimiento, preferido, siniestro, curiosa, educada...'.

La bibliografía ha vinculado el desarrollo de la capacidad intersubjetiva con el uso de este tipo de términos en el discurso adulto dirigido a los niños en edades tempranas [53-54], y ha prestado especial atención a la importancia de los textos narrativos [14, 28,55,56]; efectivamente, los cuentos infantiles facilitan al niño la adquisición de las capacidades que le permiten comprender cómo los estados mentales regulan las interacciones personales.

Para comunicarnos eficazmente y desarrollar habilidades sociales y afectivas, resulta imprescindible tener en cuenta los posibles estados mentales del interlocutor, adelantarse a su posible recepción de nuestro mensaje e, incluso, considerar la posibilidad de representaciones disociadas de la realidad (ironías, metáforas, sentidos figurados en general). En la dimensión expresiva, referir este tipo de acciones y estados es la manifestación más explícita de la capacidad intersubjetiva del hablante. Algunos de nuestros textos reflejan explícitamente las capacidades típicas requeridas por las tareas de falsa creencia: 'Jaki perseguía a una mariposa, de repente él creía que era la mariposa, pero era Senda el indio, y él se acercaba a la mariposa, creía que era ella' (C449, 9:11, F). En ciertos casos, el narrador puede intervenir señalando precisamente lo que el personaje ignora; se trata de una especie de 'información privilegiada' que procede precisamente del papel textual de narrador y que supone un paso más en la habilidad narrativa del niño y su dominio sobre el universo textual que va creando: 'Pero lo que no sabían es que una bruja los controlaba, entonces a la noche mandó que mataran a los terrestres. Supermán quedó completamente indefenso y fue presa fácil, el resto de voluntarios también, incluso los más ricos no pudieron hacer que su sueño se hiciera realidad.' (C552, 10:10, M).

Nuestros datos indican que los niños con TDAH utilizan menos estas marcas de capacidades mentalistas, apareciendo una media de 3,3 marcas en cada texto, frente a 6,8 marcas en los textos del GRC.

\section{Argumentaciones mentalistas}

Aquí ya no incluimos unidades del léxico verbal o nominal, es decir, palabras concretas, sino estructuras sintácticas causales o consecutivas que permiten al niño introducir explicaciones y justificaciones referidas a la propia escritura; en el plano formal, estas argumentaciones suelen introducirse mediante conectores del tipo 'porque', 'ya que', etc. Por ejemplo, al decir una oración como 'si tienes sed, hay cerveza en la nevera', resulta evidente que 'si tienes sed' no expresa ninguna condición para que la cerveza esté en la nevera, sino que se refiere a una justificación de la información dada: 'te lo digo por si tienes sed'.

En los textos narrativos, tales argumentaciones son una clara manifestación de la separación entre el sujeto de la enunciación (el narrador del texto) y los sujetos del enunciado (los personajes): 'Yo le pregunté qué tenían de especial esas zapatillas, y ella me contestó: Estas zapatillas de deporte son mágicas, porque se convertían en patines especiales. A la vez me encontré con Supermán que por cierto era muy simpático' (C411, 9:11, M).

Se trata de un recurso muy poco utilizado por los niños con TDAH (un 9\% de nuestros textos, frente a un $34 \%$ en el GRC, con mayor frecuencia en los textos argumentativos que en los narrativos).

\section{Marcas de polifonía textual (voces del texto)}

El tercer tipo de marca explícita de intersubjetividad corresponde a las manifestaciones directas de que el niño incluye en su texto referencias a la propia situación comunicativa o a otras interacciones y textos. En lingüística utilizamos el concepto de 'polifonía textual' [57,58] para referirnos a la multiplicidad de voces (enunciaciones) que puede incluir un mismo texto, entre la cuales la voz del autor o la del narrador es solo una más (y no siempre coincide). En este sentido, la inclusión de diálogos y voces ajenas en la redacción puede considerarse un indicio claro de conciencia metadiscursiva por parte del niño que escribe.

En primer lugar, hablamos de emergencias enunciativas para referirnos a los casos en que los protagonistas del acto comunicativo (lector y escritor) 'emergen' en el texto, casi siempre mediante el uso de la primera y la segunda persona: su aparición es más frecuente en los textos argumentativos, debido en parte a que la tarea más utilizada ('normas') se plantea como alocución a una segunda persona. Así, una argumentación sin emergencias enunciativas se caracteriza por el distanciamiento del hablante, por ejemplo: 'El niño del medio está haciendo mal y actuando mal porque está empujando, apartando, pisoteando y dando codazos, y eso es ser mala persona porque está maltratando a la gente y eso está muy mal y es una falta de respeto' (T019, 10:01, M). Por el contrario, la misma argumentación puede realizarse manifestando una implicación directa de los hablantes: 'Esta actuación es muy incorrecta. Es como si tú le pegaras a él y él no te ha hecho nada. Por ir delante no tienes que ir pegando a la gente que vaya por medio' (T002, 10:04, M). 


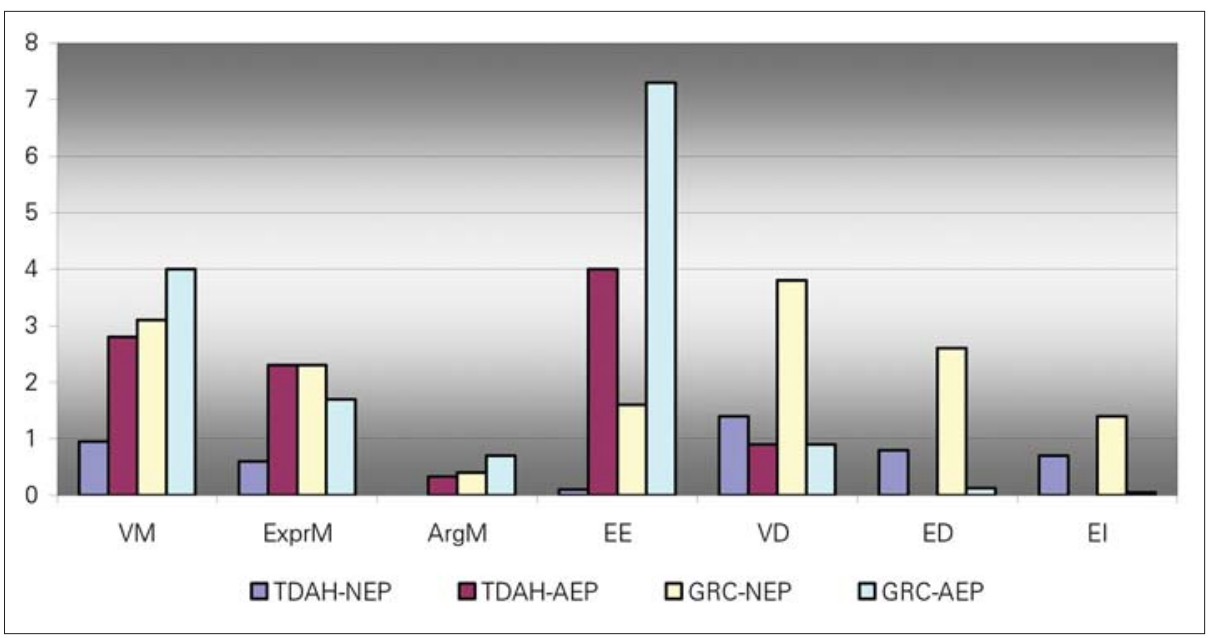

Figura. 2. Uso de marcas explícitas de intersubjetividad en ambos corpus: valores medios para cada tipo de texto. AEP: argumentaciones escritas planificadas; ArgM: argumentaciones mentalistas; ED: estilo narrativo directo; EE: emergencias enunciativas; El: estilo narrativo indirecto; ExprM: expresiones nominales mentalistas; NEP: narraciones escritas planificadas; VD: verbos de dicción; VM: verbos mentalistas.

La adquisición de la categoría morfosintáctica de persona es, como sabemos [59], un proceso que comienza con una oposición inicial de la primera persona al resto indiferenciado (noyo frente a yo), y que luego introduce en la segunda categoría la distinción de segunda y tercera persona (él-ella frente a tú); gramaticalmente, la categoría de persona se basa en el contraste subjetividad/objetividad. En este sentido, las categorías morfosintácticas que tienen morfema de persona (básicamente los verbos conjugados, pero también los deícticos del tipo 'aquíahí-allí', 'este-ese-aquel', o distinciones del tipo 'traer-llevar', 'ir-venir', etc.) pueden considerarse huella expresiva, enunciativa, de la conciencia metadiscursiva (algo que resulta pertinente en las discusiones sobre anterioridad/posterioridad entre lenguaje y capacidad intersubjetiva, versión moderna y actualizada de la polémica clásica pensamiento/lenguaje).

Los niños con TDAH de nuestra muestra utilizan también menos marcas enunciativas de este tipo; en los textos argumentativos del GRC encontramos una media de ocho emergencias enunciativas por texto, frente a una media de 3,7 en los textos AEP del grupo TDAH.

Por lo que se refiere a las emergencias enunciativas en los textos narrativos, el narrador utiliza este mecanismo discursivo para incluir alusiones al lector: 'Bien, os describiré a la princesa' (C432, 10:01, F); 'Érase una vez un pescador que estaba durmiendo. Tuvo un sueño muy raro. Te lo voy a contar. El pescador se fue navegando hasta una isla en la que vivía una bruja muy mala' (T037, 9:06, M). Frente a los textos AEP, las narraciones utilizan este recurso en mucha menor medida, posiblemente porque la posición de narrador omnisciente (en tercera persona) es mucho más conocida para los niños de nuestro entorno cultural, mientras resulta verosímil pensar que la argumentación les llega con más frecuencia en segunda persona; los niños del GRC incluyen una media de 1,5 marcas en cada texto narrativo, cifra que en el grupo TDAH se reduce a 0,1 marcas.

La segunda marca de polifonía textual corresponde a la utilización de verbos de dicción (VD), que introducen diálogos y expresiones de los personajes del texto; estos VD pueden incorporar matices añadidos relacionados con la capacidad mentalista: 'confirmar', aun teniendo valor representativo, supone reconocer veracidad en lo dicho; 'retar' traslada el peso de la acción al oyente y cuestiona su capacidad ejecutiva; 'insultar', 'agradecer' o 'maldecir' encierran información sobre el valor moral de lo dicho; 'amenazar' se carga de fuerza compromisoria, etc.

Los textos argumentativos muestran una utilización muy escasa de estos elementos para ambos grupos: aparecen en un $16,6 \%$ de los textos del corpus TDAH y un $38,7 \%$ del GRC. En ocasiones, el niño ha propuesto como norma una acción de este tipo: 'No hay que hablar todos a la vez, porque si hablamos todos a la misma vez no nos enteramos de lo que estamos diciendo, hay que respetar turnos' (T005, 11:11, M); 'No hay que insultar a la gente porque si todos insultáramos esto sería un mundo de maleducados' (C458, 9:07, F). En otros casos, la acción verbal se utiliza para las justificaciones de la norma: 'Si hay alguien que no está capacitado para hacer todas las cosas, lo menos que puedes hacer es ayudarle y así él te podrá dar las gracias y tú ser educado' (C614, 11:07, F).

En los textos narrativos, el uso de los VD es, obviamente, mucho más significativo; los VD sirven para estructurar las voces del relato, por lo que son un claro indicio de capacidad intersubjetiva. Los utiliza un $60 \%$ de los textos TDAH y un $84 \%$ de los textos del GRC. Además de utilizar los VD, los narradores incluyen en su texto el contenido de los diálogos incrustados; para ello pueden recurrir al estilo directo o al indirecto: 'Érase una vez una joven princesa que salió a pasear con su mago, le dijo la princesa al mago, llévame a la cima de la montaña' (T007, 10:05,M); 'Se encontraron una pócima, y dijo el pescador: ¡Viva, una pócima para hacerse invisible!' (T037, 9.06, M), 'El zorro le contó todo y se hicieron amigos. El karateka le dijo que él le podía ayudar y el karateka le enseñó algunas cosas para ser más fuerte' (T034, 10:01, F).

En el subgrupo de niños que utilizan los verbos de dicción, hemos considerado también el recurso al estilo directo e indirecto; entre las narraciones del grupo TDAH encontramos que un $40 \%$ de los niños reproduce literalmente lo que dicen sus personajes, y un $35 \%$ utiliza también el recurso al estilo indirecto. Las cifras vuelven a ser mayores en el GRC, donde la proporción es de un $65 \%$ de niños que usa el estilo directo, y un $75 \%$ de ellos que recurre al estilo indirecto.

A partir de este análisis, podemos establecer para cada uno de los tipos de texto valores medios previsibles de utilización de las marcas de intersubjetividad (Fig. 2).

\section{CONCLUSIONES}

En definitiva, utilizando la teoría de la enunciación hemos identificado tres marcas lingüísticas que pueden considerarse huella formal de la existencia de una capacidad intersubjetiva desarrollada, y hemos comparado su utilización por parte de niños con TDAH-GRC. Los datos nos muestran que los niños con este diagnóstico utilizan menos estas marcas que los referentes del 
GRC, tanto en los formatos narrativos como argumentativos. En concreto, las marcas identificadas son:

- Léxico específico que alude a acciones, cualidades y estados relacionados con la cognición, los sentimientos o la intención propia y/o ajena. En el GRC encontramos estas marcas en 50 de los 62 textos AEP (un $80,6 \%$, con una media de siete marcas por texto) y en 72 de los 76 textos NEP (un 94,7\%, con una media de cinco marcas por texto). Para el grupo TDAH, estas unidades léxicas mentalistas aparecen en un $100 \%$ de los textos AEP y un $55 \%$ de los textos NEP, con una media de 2,8 marcas por texto en ambos casos.

- Argumentaciones mentalistas implícitas en el texto, referidas generalmente a la actividad enunciativa o que justifican las acciones de los personajes. El GRC utiliza estas marcas en un $34 \%$ de los textos, mientras que el grupo TDAH las incluye en el 9,3\% de los textos (ningún caso en texto narrativo).

- Marcas de polifonía textual:

a) Emergencias enunciativas de escritor y lector: los textos del GRC muestran una media de ocho marcas enunciativas en cada texto argumentativo y 1,5 marcas en cada texto narrativo; sólo tres de los 138 textos prescinden de este recurso lingüístico (un 2,1\%). Por el contrario, en el grupo TDAH encontramos unos valores medios de 3,7 marcas en los textos argumentativos y 0,1 marcas en los narrativos; 23 de los 32 textos prescinden de este recurso (un $71,8 \%$ ).

b) Introducción de diálogos en el texto (verbos de dicción, mensajes en estilo directo e indirecto): los textos narrativos son los más idóneos para el despliegue de este recurso, que aparece en el $60 \%$ de los textos TDAH y el $84 \%$

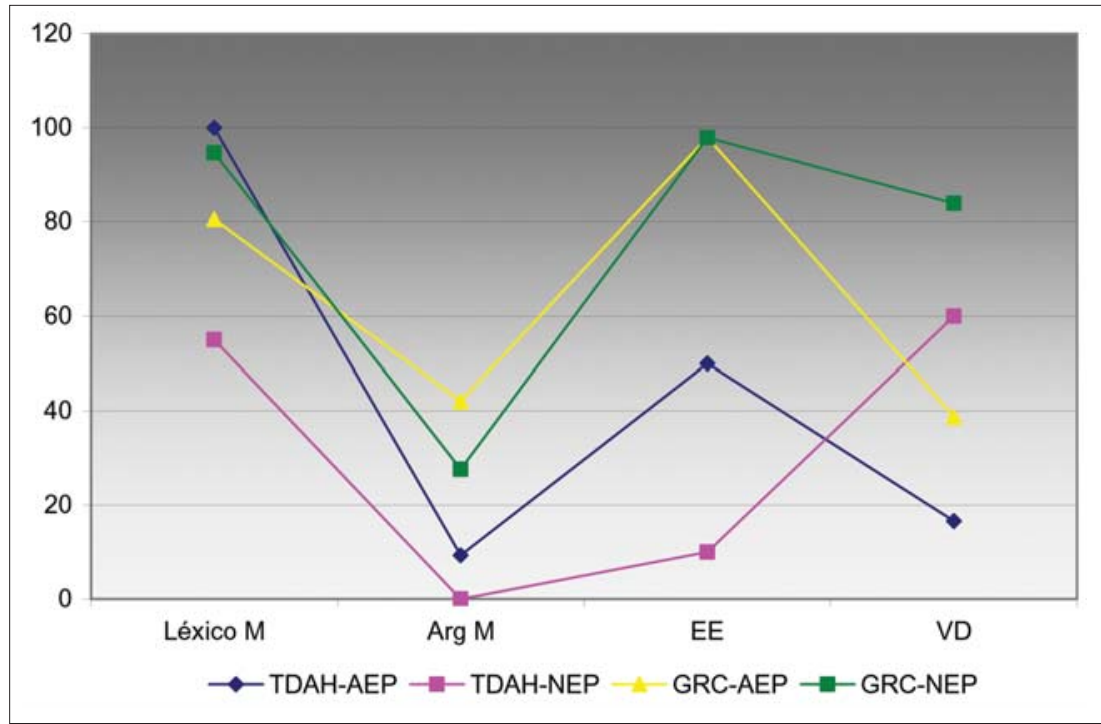

Figura. 3. Utilización de las marcas de intersubjetividad por grupos. AEP: argumentaciones escritas planificadas; Arg M: argumentaciones mentalistas; EE: emergencias enunciativas; NEP: narraciones escritas planificadas; Léxico M: léxico mentalista; VD: verbos de dicción.

de los textos del GRC. En los textos argumentativos, encontramos el uso de verbos de dicción y/o diálogos incrustados en el $16,6 \%$ de los textos del grupo TDAH y el $38,7 \%$ de los textos del GRC (Fig. 3).

Creemos que, pese a las lógicas precauciones derivadas del tamaño de la muestra, resulta plausible utilizar este tipo de análisis para desarrollar un protocolo de evaluación rápida de la capacidad intersubjetiva que complete las habituales pruebas centradas en las habilidades de comprensión. Por otro lado, la consideración de dos tipos de estructura textual, narrativa y argumentativa, nos permite comprobar la utilización selectiva de ciertos recursos, lo que confirma la necesidad de que las evaluaciones del lenguaje sean globales y no se limiten a tareas relacionadas con esquemas narrativos.

\section{BIBLIOGRAFÍA}

1. Premack D, Woodruff G. Does the chimpanzee have a theory of mind? Behav Brain Sci 1978; 1: 515-26.

2. Piaget J. El nacimiento de la inteligencia en el niño. Revista de Pedagogía 1926; 5/60: 526-36.

3. Vigotsky LS. Thinking and speech. In Rieber RW, Carton AS, eds. The collected works of L.S. Vigotsky. Vol. I. Problems of general psychology. New York: Plenum Press; 1987. p. 39-285.

4. Bruner J. Toward a theory of instruction. Cambridge, Belknap: Harvard; 1975.

5. Bruner JS. Realidad mental y mundos posibles. Los actos de la imaginación que dan sentido a la experiencia. Barcelona: Gedisa; 1988.

6. Tirapu-Ustárroz J, Pérez-Sayes G, Erekatxo-Bilbao M, Pelegrín-Valero C. ¿Qué es la teoría de la mente? Rev Neurol 2007; 44: 479-89.

7. Wimmer H, Perner J. Beliefs about beliefs: representation and constraining function of wrong beliefs in young children's understanding of deception. Cognition 1983; 13: 103-28.

8. Perner J, Leekam S, Wimmer H. Three-years-olds' difficulty with false belief. Br J Dev Psychol 1994; 5: 125-37.

9. Baron-Cohen S, Leslie AM, Frith U. Does the autistic child have a 'theory of mind'? Cognition 1985; 21: 37-46.

10. Frith U, Happé FG. Autism: beyond 'theory of mind'. Cognition 1994; 50: 115-32.

11. Leslie AM, Frith U. Autistic children's understanding of seeing, knowing and believing. Br J Dev Psychol 1998; 4: 315-24.

12. Carlson SM, Moses LJ, Hix HR. The role of inhibitory control in young children's difficulties with deception and false belief. Child Dev 1998; 9: 672-91.

13. Perner J, Leekam S, Wimmer H. Three-years-olds' difficulty with false belief. Br J Dev Psychol 1994; 5: 125-37.

14. Cassidy K, Ball L, Rourke MT, Werner RS, Feeny N, Chu JY, et al. Theory of mind concepts in children's literature. Appl Psycholinguist 1998; 19: 463-70.

15. Buitelaar JK, Van der Wees M, Swaab-Barneveld H, Van der Gaag RJ. Theory of mind and emotion-recognition functioning in autistic spectrum disorders and in psychiatric control and normal children. Dev Psychopathol 1999; 11: 39-58.

16. Peterson CC, Siegal M. Insights into theory of mind from deafness and autism. Mind Lang 2000; 15: 123-45.

17. Tager-Flusberg H. Language and understanding minds: connections in autism. In Baron-Cohen S, Tager-Flusberg H, Cohen DJ, eds. Understanding other minds: perspectives from autism and developmental cognitive neuroscience. Oxford: Oxford University Press; 2000. p. 124-49.

18. Surian L, Siegal M. Sources of performance on theory of mind tasks in right hemisphere-damaged patients. Br Lang 2001; 78: 224-32.

19. Joseph RM, Tager-Flusberg H. The relationship of theory of mind and executive functions to symptom type and severity in children with autism. Dev Psychopathol 2004; 16: 137-55.

20. Gallagher HL, Frith CD. Functional imaging of 'theory of mind'. Trends Cogn Sci 2003; 7: 77-83. 
21. Scholl BJ, Leslie AM. Minds, modules, and meta-analysis. Child Dev 2001; 72: 696-701.

22. Hobson RP. El autismo y el desarrollo de la mente. Madrid: Alianza; 1991.

23. Rondal J. Teoría de la mente y lenguaje. Rev Logop Fon Audiol 2007; 27: 51-5.

24. Benveniste E. Problemas de lingüística general, II. México: Siglo XxI; 1977; p. 82-91.

25. Gallardo-Paúls B. Más allá de las palabras y su estructura: las categorías del componente pragmático. In Garayzábal E, ed. Lingüística clínica y logopedia. Madrid: Antonio Machado; 2006. p. 81-196.

26. Gallardo-Paúls B. Categorías inferenciales en pragmática clínica. Rev Neurol 2005; 41: 65-71.

27. Wellman HM, Cross D, Watson J. Meta-analysis of theory-of-mind development: the truth about false belief. Child Dev 2001; 72: 655-84.

28. Happé FG. An advanced test of theory of mind: understanding of story characters' thoughts and feelings by able autistic, mentally handicapped, and normal children and adults. J Autism Dev Disord 1994; 24: $129-54$

29. Fletcher PC, Happé F, Frith U, Baker SC, Dolan RJ, Frackowiak RS, et al. Other minds in the brain: a functional imaging study of "theory of mind' in story comprehension. Cognition 1995; 57: 109-28.

30. Happé F, Brownell H, Winner E. Acquired 'theory of mind' impairments following stroke. Cognition 1999; 70: 211-40.

31. Winner E, Brownell H, Happé F, Blum A, Pincus D. Distinguishing lies from jokes: theory of mind deficits and discourse interpretation in right hemisphere brain-damaged patients. Brain Lang 1998; 62: 89-106.

32. Baron-Cohen S, O'Riordan M, Stone V, Jones R, Plaisted K. Recognition of faux pas by normally developing children and children with Asperger syndrome or high-functioning autism. J Autism Dev Disord 1999; 29: 407-18.

33. Olson DR. On the origins of beliefs and other intentional states in children. In Astington JW, Harris PL, Olson DR, eds. Developing theories of mind. New York: Cambridge University Press; 1988. p. 414-26.

34. Nelson K. Language in cognitive development: the emergence of the mediated mind. New York: Cambridge University Press; 1996.

35. Garfield J. Social cognition, language acquisition and the development of the theory of mind. Mind Lang 2001; 16: 494-541.

36. Bruner JS. Explaining and interpreting: two ways of using mind. In Harman G, ed. Conceptions of the human mind: essays in honor of George A. Miller. Hillsdale, NJ: Lawrence Erlbaum; 1993. p. 123-36.

37. Baker L, Cantwell D. Attention deficit disorder and speech/language disorders. Comprehensive Mental Health Care 1992; 2: 3-16.

38. Merritt DD, Liles BZ. Story grammar ability in children with and without language disorder: story generation, story retelling, and story comprehension. J Speech Hear Res 1987; 30: 539-52.

39. Tannock R, Purvis K, Schachar R. Narrative abilities in children with attention deficit hyperactivity disorder and normal peers. J Abnorm Child Psychol 1993; 21: 103-17.

40. Liles BZ, Duffy RJ, Merritt DD, Purcell SL. Measurement of narrative discourse ability in children with language disorders. J Speech Hear Res 1995; 38: 415-25.
41. Pérez-Pérez E. Cohesión y coherencia en las narraciones de niños y niñas con trastorno específico del lenguaje. Rev Logop Fon Audiol 1997; 17: 103-11.

42. Lorch E, Diener M, Sánchez R, Milich R, Welsh R, Van den Broek P. The effects of story structure on the recall of stories in children with attention deficit hyperactivity disorder. J Educ Psychol 1999; 91: 273-83.

43. Kaderavek JN, Sulzby E. Narrative production by children with and without specific language impairment: oral narratives and emergent readings. J Speech Hear Res 2000; 43: 34-49.

44. Renz K, Lorch EP, Milich R, Lemberger C, Bodner A, Welsh R. Online story representation in boys with attention deficit hyperactivity disorder. J Abnorm Child Psychol 2003; 31: 93-104.

45. Parigger E, Baker A, De Jong J. Narrative abilities in children with attention deficit hyperactivity disorder and normally developing children [poster]. Presentation at the University of Newcastle upon Tyne, Child Language Seminar, July, 19-21, 2006.

46. Gillig JM. El cuento en pedagogía y en reeducación. Madrid: Fondo de Cultura Económica; 2000.

47. Vaquerizo-Madrid J, Estévez-Díaz F, Pozo-García A. El lenguaje en el trastorno por déficit de atención con hiperactividad: competencias narrativas. Rev Neurol 2005; 41: 83-9.

48. Gallardo-Paúls B. Pragmática para logopedas. Cádiz: Publicaciones de la Universidad de Cádiz; 2007.

49. Miranda-Casas A, Ygual-Fernández A, Rosel J. Complejidad gramatical y mecanismos de cohesión en la pragmática comunicativa de los niños con trastorno por déficit de atención con hiperactividad. Rev Neurol 2004; 38: 111-6.

50. Brown J, Donelan-McCall N, Dunn, J. Why talk about mental states? The significance of children's conversations with friends, siblings, and mothers. Child Dev 1996; 67: 836-49.

51. Slade L, Ruffman T. Parental use of emotional and mental state terms to their children in conflict situations and theory of mind development: a longitudinal study. BPS Developmental Section Annual Conference, Nottingham University, 6-9 September 2000.

52. Fernández-Pérez M. Dinamismo construccional en el lenguaje infantil y teoría lingüística. ELUA 2003; 17: 273-87.

53. MacLean M, Moore B, Carter W. Children's use of mental state terms. Proceedings of BPS Annual Conference 2000.

54. Symons DK, Peterson CC, Slaughter V, Roche J, Doyle E. Theory of mind and mental state discourse during book reading and story-retelling tasks. Br J Dev Psychol 2005; 23: 1-24.

55. Astington JW. The child's discovery of the mind. Cambridge: Harvard University Press; 1993.

56. Rabazo-Méndez MJ, Moreno-Manso JM. Teoría de la mente: la construcción de la mente mediante los cuentos de hadas. Enseñanza e Investigación en Psicología 2007; 12: 179-201.

57. Tannen D. Talking voices. Repetition, dialogue, and imagery in conversational discourse. Cambridge: Cambridge University Press; 1989.

58. Kristeva J. The system of the speaking subject. In Moi T, ed. The Kristeva reader. Oxford: Basil Blackwell; 1986: 34-61.

59. Serra M, Serrat E, Solé R, Bel A, Aparici M. La adquisición del lenguaje. Barcelona: Ariel; 2000.

\section{THE LINGUISTIC MARKS IN THE THEORY OF MIND: INTERSUBJECTIVITY AND ENUNCIATION IN ATTENTION DEFICIT HYPERACTIVITY DISORDER}

Summary. Introduction. The tests that are commonly used to analyse whether a subject has developed mentalistic and intersubjective capacities (theory of mind) are restricted to tasks that assess comprehension, without taking into account the active, expressive marks of those capacities. Development. Taking Benveniste's pragmatic theory of enunciation as our framework, we analyse texts written by children who have been diagnosed with attention deficit hyperactivity disorder $(A D H D)$ in order to confirm the presence of formal marks of enunciation. We distinguish between the explicit marks in a text (verb or noun phrases that refer to mental states and also diction) and marks embedded within the text (dialogues, allusions to other texts, textual polyphony). The corpus of data used for this analysis consisted of 170 narrative and argumentative texts written by children aged between 9 and 11 years, of whom those who had been diagnosed as suffering from combined-type ADHD constituted a subgroup. Conclusions. The theory of enunciation provides a suitable framework for studying the expressive, active manifestation of the intersubjective capacity of children. [REV NEUROL 2008; 46 (Supl 1): S29-35] Key words. Attention deficit hyperactivity disorder. Linguistic deficit. Narration and argumentation. Textual pragmatics. Theory of mind. 